\title{
Évaluation de l'exposition d'adipocytes humains sous-cutanés en culture aux acides linoléiques conjugués par une approche multi-omique
}

\author{
Jean-Charles MARTIN ${ }^{1}$ \\ Karima BENCHARIF ${ }^{1}$ \\ Bruno BERTHET ${ }^{2}$ \\ Nathalie BANZET ${ }^{1}$ \\ Romain $\mathrm{BOTT}^{1}$ \\ Catherine DEFOORT ${ }^{1}$ \\ Marie-Christine ALESSI ${ }^{3}$ \\ 1 UMR Inra 1260/Inserm 1025/ \\ Universités Aix-Marseille I \& II, \\ Nutriments lipidiques \& prévention \\ des maladies métaboliques, \\ Faculté de médecine de La Timone, \\ 27, Bvd Jean Moulin, \\ 13385 Marseille, \\ France \\ $<$ jean-charles.martin@univmed.fr $>$ \\ ${ }^{2}$ Hôpital de la Timone Adultes, \\ Chirurgie viscérale et générale, \\ SCE Chirurgie générale et digestive, \\ 13385 Marseille \\ 3 Inserm 626, \\ Laboratoire d'hématologie, \\ syndrome métabolique, \\ tissu adipeux et risque vasculaire, \\ Faculté de Médecine La Timone, \\ 13385 Marseille
}

\begin{abstract}
Conjugated linoleic acids are 18 carbones fatty acids members comprising a consecutive (conjugated) double bonds system with a various cis/trans geometry sequence, giving rise to at least 16 different isomers. Among those, the t10,c12 isomer has been reported to have anti-obesity properties. We evaluated the biological response of human primary adipose tissue cultured adipocytes to that CLA isomer, alone or present in a commercial mixture, using system biology approaches (primarily transcriptomics and metabolomics). We found that CLA changed the biological activity of at least 45 metabolic pathways at the genomic level, which transcriptional activities was associated to a parallelle metabolome adipocyte change (lipidome upmost) (r2value transcriptome) metabolome of 0.89). In pairwise analysis, it appeared that 6 of these pathways at the genomic level were tightly associated to the metabolomic response, such as apoptosis, interleukine-6, proteasome, reticulum endoplasmic stress, transcription role of heterochromatine, cell proliferation through EGFR dependent tyrosine kinase, gamma-aminobutyrate receptor. This latter exhibited the most tightly relationship with the metabolome variations in multivariated analysis. One may infer that this pathway is the most targeted by CLA treatment. In conclusion, the implementation of a multi-omic global approach allowed the identification of a set of biological pathways at the genomic level associated to the metabolic balance of CLA-treated adipocytes. All of these pathways are related to insulin-resistance, metabolic syndrome and adipogenesis.
\end{abstract}

Key words: subcutaneous adipocytes, obesity, conjugated linoleic acid, metabolomics, transcriptomics

\section{Introduction}

Les CLA font l'objet d'une recherche intense depuis le début des années 1990, notamment pour évaluer les modalités de leur effet anti-obésité (Roche et al., 2001). Si cette activité est bien documentée et avérée dans certains modèles animaux (souris (West et al., 1998), hamsters (Navarro et al., 2009), rats génétiquement obèses (Kelley et Erickson, 2003), en revanche les effets sont plus ténus et controversés chez I'humain (Whigham et al., 2007). Certains pointent un risque d'aggraver le syndrome métabolique chez des sujets consommant des préparations contenant l'isomère $\mathrm{t} 10, \mathrm{c} 12$ et chez qui ce syndrome préexiste (Riserus et al., 2002). Finalement, plus que son efficacité vis-à-vis de la réduction de la masse grasse, le point prépondérant devient la question de l'absence d'effets délétères. Nous avons choisi d'évaluer le retentissement biologique de la consommation de CLA contenant I'isomère t10,c12 sur des adipocytes humains sous-cutanés en culture, le tissu adipeux étant une cible majeure pour les CLA dans le cadre des effets anti-obésité et d'insulino-résistance.

Pour citer cet article : Martin JC, Bencharif K, Berthet B, Banzet N, Bott R, Defoort C, Alessi MC. Évaluation de l'exposition d'adipocytes humains sous-cutanés en culture aux acides linoléiques conjugués par une approche multi-omique. OCL $2011 ; 18(6)$ : 365-371. doi : 10.1684/ocl.2011.0413 


\section{Matériel et méthode}

Notre approche a consisté à mesurer de la façon la plus exhaustive possible la réponse aux traitements par une analyse transcriptomique par biopuce couplée à une analyse métabolomique réalisée par spectrométrie de masse (figure 1). Cette stratégie a permis de sélectionner les réponses transcriptomiques qui donnaient lieu à une réponse fonctionnelle métabolique. Six cultures initiales d'adipocytes ont été divisées en 3 lots pour être incubées pendant 48 heures par $(50 \mu \mathrm{M}$ chacun sous forme d'acide gras libre lié à l'albumine) I'acide linoléique, I'isomère t10,c12 ou un mélange commercial de CLA enrichi à $90 \%$ avec l'isomère $\mathrm{c} 9, \mathrm{t} 11$ et $\mathrm{t} 10$, c12. La mesure de l'expression des gènes de ces cultures a été réalisée sur des biopuces pangénomiques Agilent 43k. Le profilage métabolique a été accompli par spectrométrie de masse sur extraits cellulaires polaires et non-polaires. Après extraction et ajustement informatique des signaux et curation, les données ont été traitées avec des méthodes d'analyses multivariées en mode PLS principalement (figure 2). Une première analyse a permis d'obtenir 1359 gènes, 80 signaux métaboliques non polaires et 23 polaires qui étaient sensibles aux traitements (taux de classification globale pour la mesure de la spécificité des traitements de 94,12\%). De façon intéressante, les variables lipidiques étaient dans I'ensemble diminuées par les traitements aux CLA comparativement au traitement avec I'acide linoléique. À ce stade, même réduite l'abondance des variables biologiques affectées par les traitements ne permettait pas une interprétation compréhensible de I'information biologique. Pour faciliter celle-ci, nous avons regroupé les gènes sélectionnés en 63 voies biologiques à l'aide du logiciel d'enrichissement Metacore (figure 3). L'activité biologique de chacune d'entre elles a été quantifiée par la construction de nouvelles variables " voies biologiques". Ceci a été accompli à partir des valeurs d'expression des gènes constitutifs de chaque voie, "compressés " en une valeur unique et représentative de la voie biologique (méthode d'analyse multibloc avec construction de variables représentatives par PLS hiérarchique). Pour les métabolites, un procédé analogue a été réalisé, sauf que le métabolome n'étant pas précisément annoté, nous avons construit les nouvelles variables métaboliques sur la base de la proximité statistique des signaux métaboliques détectés (méthode de regroupement par classification hiérarchique ascendante) (figure 3). Sept ensembles métaboliques ont ainsi pu être construits et leur intensité quantifiée. Nous avons vérifié que la compression en ensembles biologiques ne modifie pas la classification des groupes de traitements en analyse discriminante (taux global de classification de 88,24\%). Notre but était de sélectionner les voies biologiques au niveau génomique qui avait la meilleure traduction au niveau métabolomique, et qui avait donc la meilleure chance d'avoir une traduction fonctionnelle.

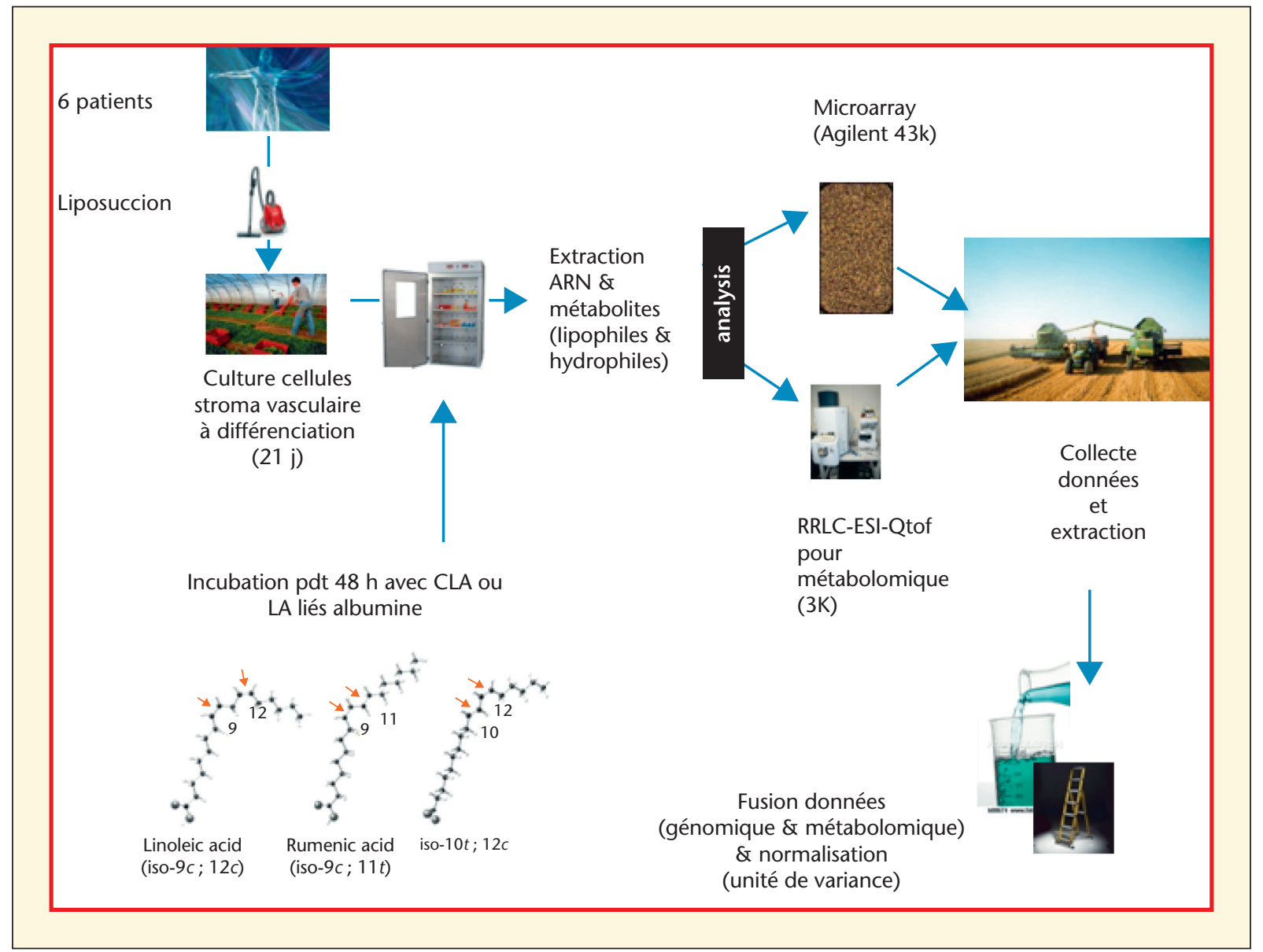

Figure 1. Schéma général du protocole utilisé pour l'analyse multi-omique des adipocytes en culture traités par différentes formes de CLA. 
Nous avons calculé un indice de détermination $\mathrm{R} 2=0,66$ entre la réponse génomique et la réponse métabolomique (analyse de régression multivariée PLS) ; après une étape supplémentaire de sélection des meilleurs prédicteurs génomiques et des meilleurs prédits métabolomique (critères VIP et R2Y/Q2Y de I'analyse PLS), nous avons retenu 45 voies biologiques au niveau génomique et 3 ensembles métaboliques qui étaient liés avec un indice de détermination R2 $=0,90$.

Ainsi, 43 voies biologiques au niveau génomique dont l'activité était modifiée par les traitements aux CLA avaient un lien statistique avec 3 ensembles métaboliques (figure 4).
Afin de mieux préciser quelles étaient les voies génomiques qui paraissaient avoir le plus d'association avec la réponse métabolomique, nous avons recherché à l'aide d'une analyse en matrice de corrélations quelles étaient les voies biologiques génomiques qui étaient directement appariées aux ensembles métabolomiques. Cette analyse est visualisée sous la forme d'un graphe d'interaction, dans lequel nous avons extrait un sous-graphe correspondant aux relations appariées entre réponse génomique et réponse métabolomique (figure 5). La superposition de l'activité globale de chaque voie biologiques en fonction des traitements indique que certains ensembles sont plus sensibles au mélange commercial

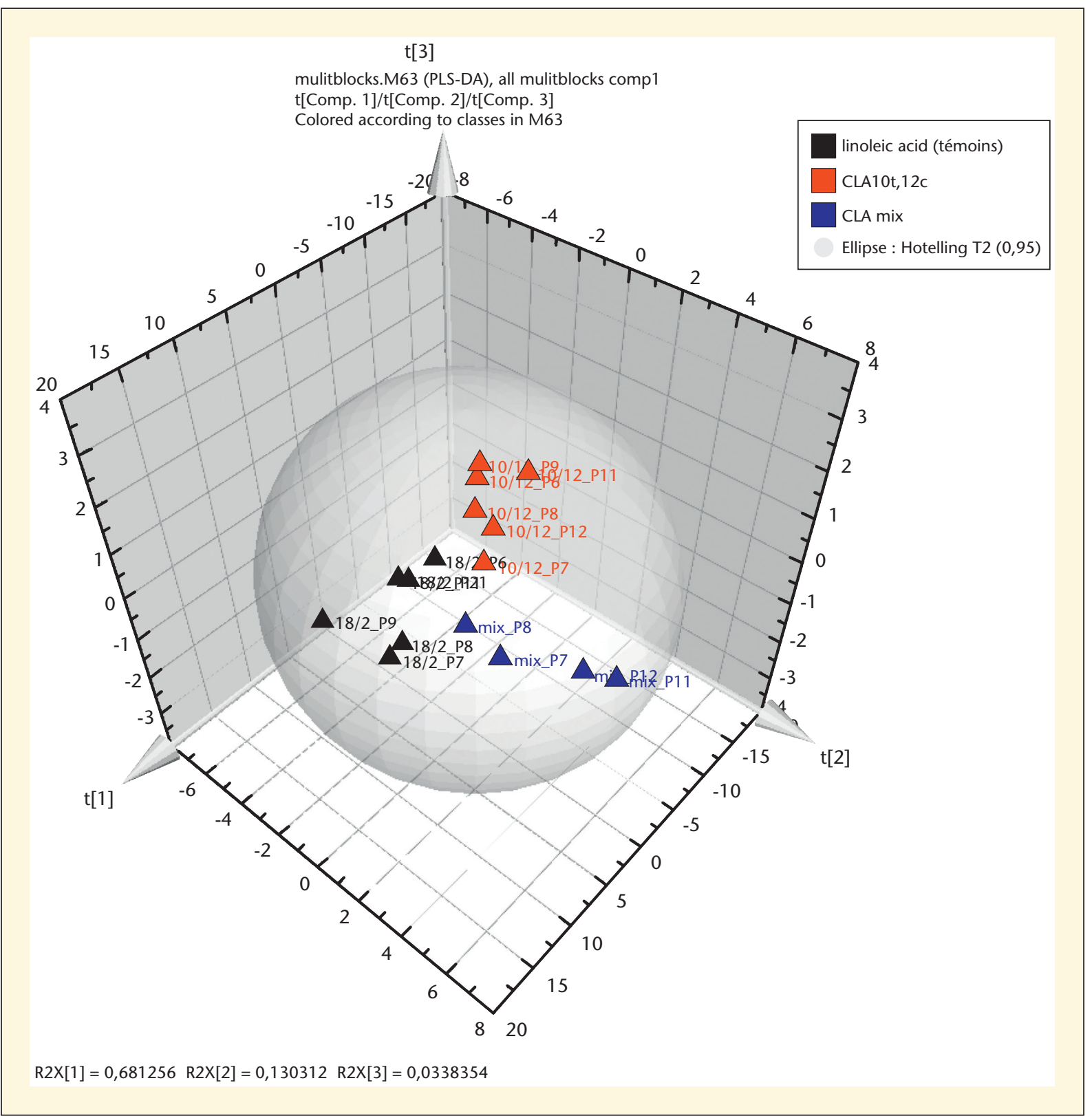

Figure 2. Graphe d'analyse discriminante en mode PLS représentant la réponse biologique multi-omique (transcriptomique et métabolomique) des adipocytes pour chacun des trois traitements : 18/2_P, traitement à l'acide linoléique ; 10/12_P, traitement avec l'isomère t10,c12_CLA ; mix_P, traitement avec le mélange commercial d'isomères de CLA. 
A

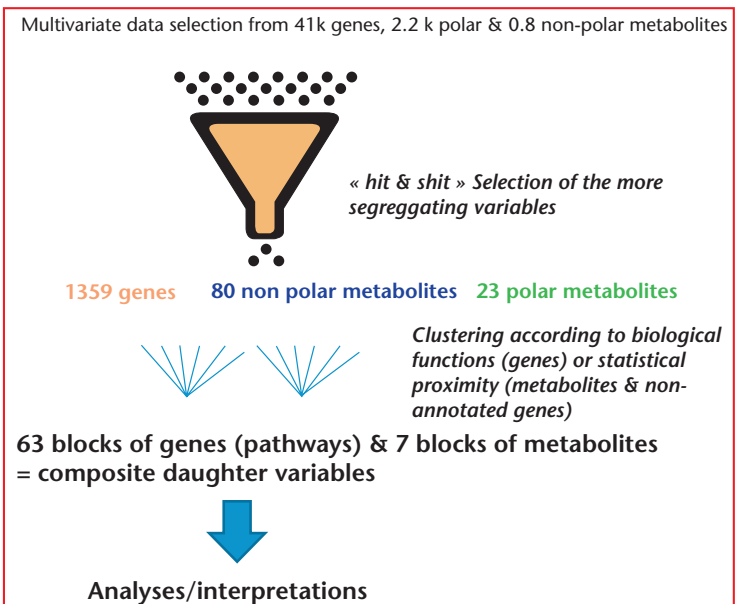

B

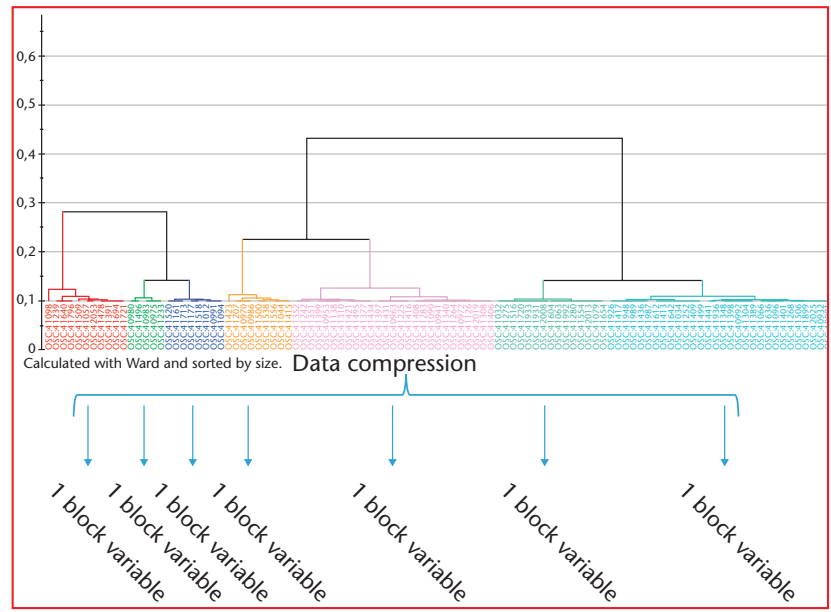

Figure 3. Procédure de regroupement des variables biologiques individuelles en ensemble biologique plus réduit par une méthode d'analyse en composante PLS hiérarchique. A) procédure générale; B) procédure de regroupement des métabolites et gènes non-annotés en fonction de leur proximité statistique

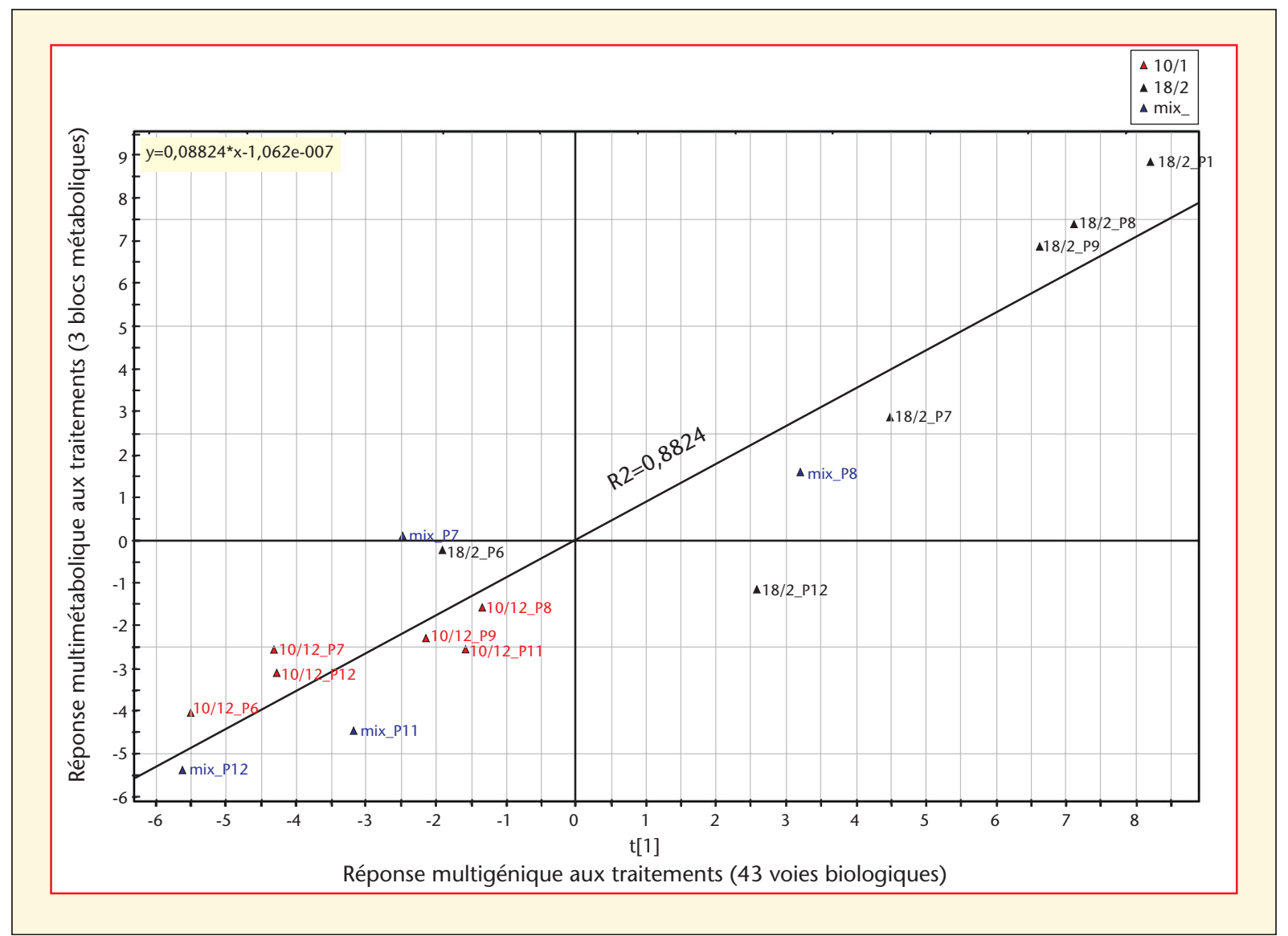

Figure 4. Analyse PLS représentant I'association entre la réponse multigénique aux traitements (43 voies biologiques identifiées au niveau génomique) et la réponse multimétabolique ( 3 blocs de métabolites) pour chaque culture d'adipocyte; $R 2=0.88, n=16$ cultures d'adipocytes différentes. 


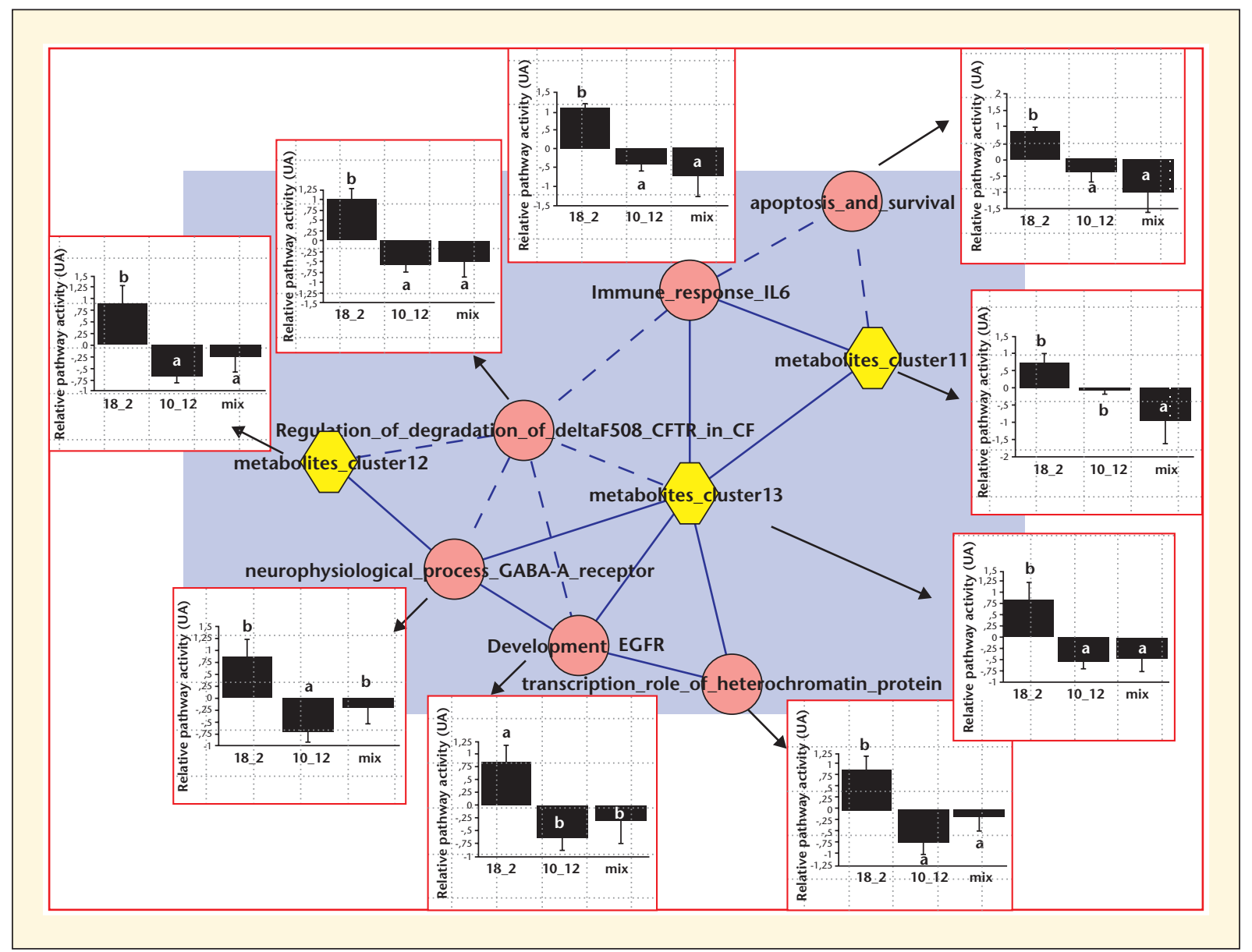

Figure 5. Effet des traitements sur l'intensité de la réponse des voies génomiques liées au métabolome cellulaire. Les valeurs d'intensité des voies biologiques correspondent aux scores composites calculés par PLS hiérarchique. Par convention, les valeurs de régulation par le traitement contrôle sont toujours représentées en valeur positive, les groupes traités étant considérés relativement aux valeurs contrôles. ${ }^{a, b, c} p<0,05$. Les liens entre les noeuds du réseau représentent des coefficients de corrélations de Spearmann (rho $>0,7$ ), soit positif (trait plein), soit négatif (trait pointillé).

de CLA, et d'autres à l'isomère t10,c12 seul (figure 5). Un examen de la littérature sur les voie métaboliques du sous-graphe indique que celles-ci sont toutes impliquées dans des phénomènes d'insulino-résistance et d'intolérance au glucose dans les phénomènes d'obésité (figure 6). La contribution calculée des facteurs génomiques à la réponse métabolomique indique que la voie du GABA-récepteur est prépondérante.

\section{Conclusion}

II apparait que l'exposition d'adipocytes humains en culture aux CLA s'accompagne de la modification de la régulation d'au moins 45 voies biologiques différentes liées à des changements du métabolome. Parmi ces 45 voies, 6 sont directement liés aux modifications du métabolome cellulaire (lipidome essentiellement) et concernent des effets biologiques liées à l'apoptose, la régulation de gènes liés à la lipodystrophie, la tolérance au glucose, l'adipogenèse et à I'insulino-résistance. Au-delà des effets déjà rapportés des CLA sur l'apoptose et l'inflammation dans le tissu adipeux, nous rapportons un impact de ces acides gras sur la transmission de neuromédiateurs et sur l'activité du protéasome. L'analyse en réseau indique la façon dont l'ensemble des phénomènes sont en interaction pour produire des changements fonctionnels (modification du métabolome). Peu de différences sont notées entre l'exposition à l'isomère t10,c12-CLA ou au mélange commercial de cet isomère avec l'isomère $c 9, \mathrm{t} 11$ CLA, ce qui confirme chez I'humain tout comme chez les modèles murins que l'isomère $t 10, c 12-C L A$ est biologiquement actif.

Nous avons pu noter une régulation inverse de voies biologiques potentiellement impliquées dans le développement du diabète de type 2 des CLA comparativement au traitement contrôle. Ceci confirme les précautions qu'il convient de prendre concernant la consommation de ce produit et ses dérivés commerciaux chez l'humain.

L'approche multi-omique sans a priori que nous avons mis en œuvre a détaillé l'ensemble des modifications qui interviennent au niveau de l'adipocyte traité aux CLA, et de sélectionner parmi les processus biologiques affectés au niveau génomique ceux qui étaient les plus liés à des modifications du métabolome. Contrairement aux approches classiques qui ne considèrent généralement les variables 


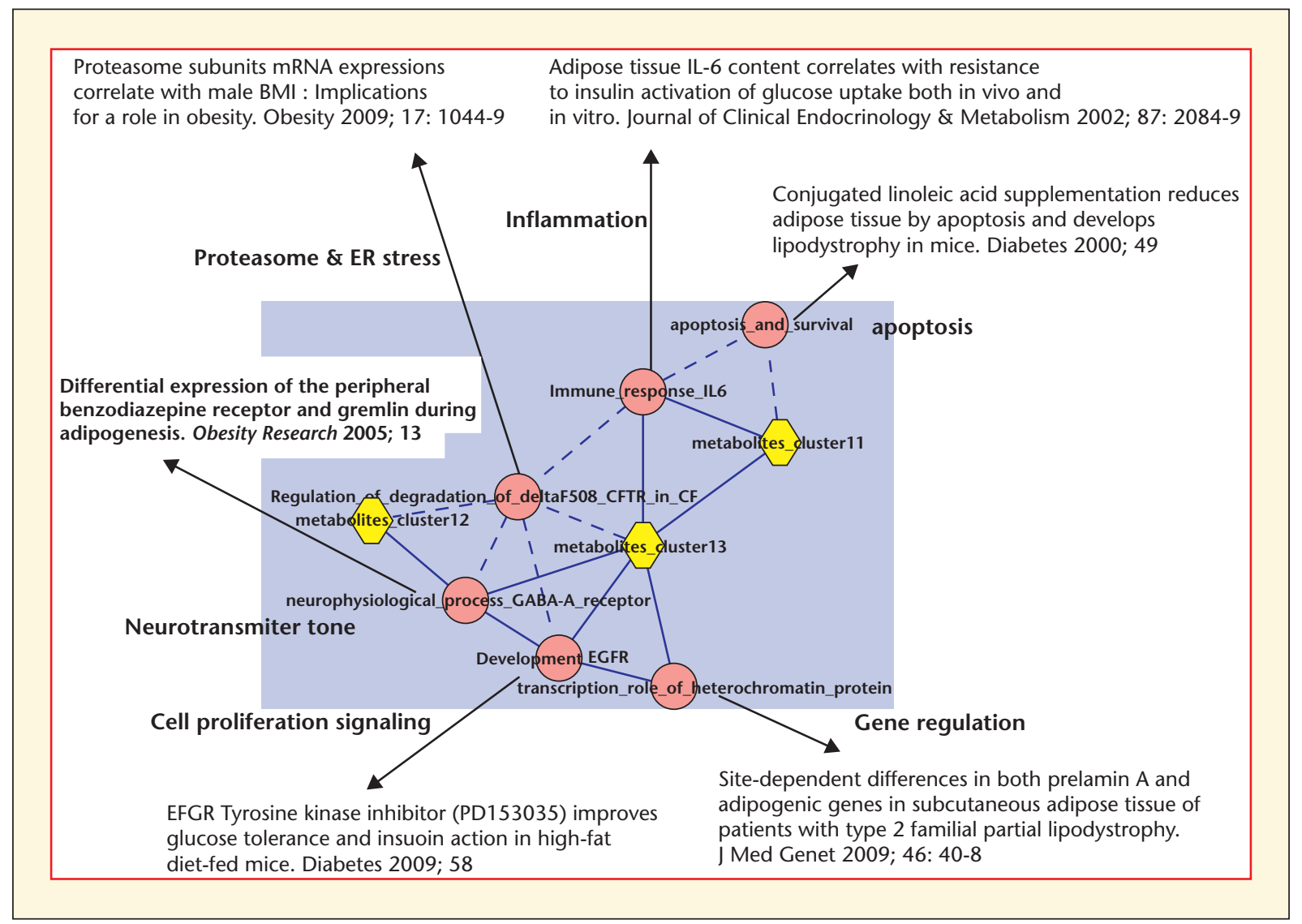

Figure 6. Conséquence fonctionnelle de l'administration de I'isomère t10,c12-CLA et d'un mélange commercial de CLA sur l'adipocyte humain culture (Prada et al., 2009 ; Sakamoto et al., 2009 ; Bastard et al., 2002 ; Tsuboyama-Kasaoka et al., 2000; Araujo-Vilar et al., 2009; Wade et al., 2005)

biologiques qu'indépendamment et individuellement, notre approche considère ces variables en ensembles biologiques ("pathway") modélisés mathématiquement et permet une représentation simple et compréhensible de phénomènes complexes. Cette modélisation est à notre sens plus proche de la réalité où les phénotypes sont déterminés plus par des modifications de voies biologiques en interaction que par des événements moléculaires isolés. Au-delà de cette étude sur les CLA, I'approche originale de génomique fonctionnelle multi-omique que nous avons développée grâce à cette étude ouvre des perspectives intéressantes pour rechercher les mécanismes moléculaires qui ont une portée sur les phénotypiques moléculaires. Nous allons compléter nos résultats par l'identification des métabolites correspondant aux masses moléculaires détectées et qui ont été affectés par les traitements, ce qui donnera des informations complémentaires sur la façon dont des ensembles de gènes peuvent retentir sur certains aspects du métabolisme cellulaire ${ }^{1}$.

\footnotetext{
${ }^{1}$ Article issu d'un projet de recherche ayant bénéficié du soutien du Groupe lipides nutrition (GIn).
}

\section{RÉFÉRENCES}

Araujo-Vilar D, Lattanzi G, Gonzalez-Mendez B, et al. Site-dependent differences in both prelamin $A$ and adipogenic genes in subcutaneous adipose tissue of patients with type 2 familial partial lipodystrophy. I Med Genet 2009 ; $46:$ 40-8.

Bastard J-P, Maachi M, van Nhieu JT, et al. Adipose Tissue IL-6 Content Correlates with Resistance to Insulin Activation of Glucose Uptake both in Vivo and in Vitro. J Clin Endocrinol Metab 2002 ; 87 : 2084-9.

Kelley DL, Erickson KL. Modulation of Body Composition and Immune CellFunctions by Conjugated Linoleic Acid in Humans and Animal Models: Benefits vs. Risks. Lipids 2003 ; 38 : 377-86.

Navarro V, Portillo MP, Margotat A, et al. A multi-gene analysis strategy identifies metabolic pathways targeted by trans-10, cis-12conjugated linoleic acid in the liver of hamsters. Br J Nutr $2009 ; 102$ : 537-45.

Prada PO, Ropelle ER, Mourao RH, et al. EGFR tyrosine kinase inhibitor (PD153035) improves glucose tolerance and insulin action in high-fat diet-fed mice. Diabetes 2009 ; 58 : 2910-9.

Riserus U, Basu S, Jovinge S, Fredrikson GN, Arnlov J, Vessby B. Supplementation with conjugated linoleic acid causes isomerdependent oxidative stress and elevated C-reactive protein: a potential link to fatty acid-induced insulin resistance. Circulation 2002 ; 106 : 1925-9. 
Roche HM, Noone E, Nugent A, Gibney MJ. Conjugated linoleic acid: a novel therapeutic nutrient? Nutr Res Rev $2001 ; 14$ : 173-87.

Sakamoto K, Sato Y, Shinka T, et al. Proteasome subunits mRNA expressions correlate with male BMI: implications for a role in obesity. Obesity (Silver Spring) $2009 ; 17: 1044-9$.

Tsuboyama-Kasaoka N, Takahashi M, Tanemura K, et al. Conjugated linoleic acid supplementation reduces adipose tissue by apoptosis and develops lipodystrophy in mice. Diabetes 2000; 49:153442.
Wade FM, Wakade C, Mahesh VB, Brann DW. Differential expression of the peripheral benzodiazepine receptor and gremlin during adipogenesis. Obes Res $2005 ; 13: 818-22$.

West DB, Delany JP, Camet PM, Blohm F, Truett AA, Scimeca J. Effects of conjugated linoleic acid on body fat and energy metabolism in the mouse. Am J Physiol 1998 ; 1998; 275: R667-72.

Whigham LD, Watras AC, Schoeller DA. Efficacy of conjugated linoleic acid for reducing fat mass: a meta-analysis in humans. Am J Clin Nutr $2007 ; 85: 1203-11$. 